\title{
New spinal cord injury standards, 1992
}

\author{
J F Ditunno Jr MD \\ Department of Rehabilitation Medicine, Thomas Jefferson University Hospital, \\ Philadelphia, Pensylvania, USA.
}

The importance of uniform standards for classification of spinal cord injury subjects has been recognized for more than 20 years, ${ }^{1}$ and has received considerable discussion recently at meetings in the United States. ${ }^{23}$ As a result, a committee of the American Spinal Injury Association (ASIA) with support principally from the American Paralysis Association (APA), and in part from ASIA and the National Institute of Disability and Rehabilitation Research (NIDRR), made up of representatives of neurological surgery, orthopaedic surgery, and physiatry was formed. With input from participants from the National Data Base of NIDRR, the National Acute Spinal Cord Injury Study (NASCIS), the National Institutes of Health (NIH), and members of the International Medical Society of Paraplegia (IMSOP), most of the organizations and agencies concerned and experienced in the use of these classifications were represented. At a meeting on September 6, 1991 in Philadelphia, USA preliminary agreement was reached on the following: key muscles for motor level classification; key points for sensory level classification; motor and sensory indices; revised definitions of neurological level; complete and incomplete lesion; zone of partial preservation; neuroanatomical syndromes; a modified Frankel classification; and a functional/disability index called the Functional Independence Measure. The new standards represent a modification of the ASIA 1989 Standards which incorporates a number of elements such as sensory indices, options for other sensory modalities to be tested, and additional recommended muscles from the NASCIS study group which allows continuity with the database of previous studies. Many of the elements of these standards will be specified below but agreement at this time is preliminary and may be further modified by additional comment. The key muscles for motor level classification are as follows: C5 elbow flexors, C6 wrist extensors, $\mathrm{C} 7$ elbow extensors, C8 finger flexors, middle distal phalanx, T1 fifth finger abductor, L2 hip flexors, L3 knee extensors, L4 ankle dorsiflexors, L5 large toe extensors, S1 ankle plantar flexors. Each muscle is graded on a scale of $0-5$. These 10 muscles would be utilized to calculate the motor index score of 100 for right and left sides. The key sensory points from the ASIA standards would be utilized for sensory level classification. Pin and touch would be scored separately on a scale of $0-2$. A sensory index could be calculated based on 28 dermatomes for a total score of 112 for both right and left sides. The neurological level would be defined as the most caudal segments which test as normal or intact for both motor and sensory function. There could be differences in level for motor or sensory and right or left. The zone of partial preservation is defined as all segments below the neurological level with partial preservation of sensory or motor findings. A complete lesion is defined as absence of sensory and motor function in the lowest sacral segment. Sacral sensation includes light touch or pain at the mucocutaneous junction of the anus. The motor function to test is the presence of voluntary contraction of the anal sphincter on manual examination. An incomplete lesion would be defined as partial preservation of motor or sensory findings below the neurological level which included the lowest sacral segment. Incomplete syndromes such as Central Cord and Brown-Séquard would be retained with a clinical description but without reference to the neuroanatomical lesion. The current modified Frankel classification would also 
be retained. A completely new classification of function/disability would be added, called the Functional Independence Measure (FIM). This scores 20 items of self care, mobility, sphincter control, locomotion, communication and social cognition on a 7 point scale for each item. Following publication of the new standards in 1992, the committee plans on the development of a more detailed manual of instructions.

Members of the committee are Drs Michael Bracken, Margaret Brown, Gra- ham Creasey, John Ditunno, William Donovan, Thomas Ducker, Frederick Maynard, Samuel Stover, Charles Tator, Robert Waters, Jack Wilberger and Wise Young. There will be an annual review of the standards, and revisions will be made based on research advances. The committee wishes to express its appreciation for the opportunity to communicate with an international audience and welcomes your comments.

\section{References}

1 Michaelis LS (1969) International inquiry on neurological terminology and prognosis in paraplegia and tetraplegia. Paraplegia 7: 1-5.

2 Conference on Spinal Cord Injury (1990) Neurological and Functional Recovery. National Rehabilitation Research and Training Center in Spinal Cord Injury, Jefferson Medical College of Thomas Jefferson University, Philadelphia, PA.

3 American Spinal Injury Association (1990) Sixteenth annual scientific meeting, Orlando, Florida.

4 American Spinal Injury Association (1989) Standards for Neurological Classification of Spinal Injury Patients. Chicago. 